\title{
Cardiac Tolerability of Concurrent Administration of Trastuzumab and Anthracycline-Based Regimen as Adjuvant Chemotherapy for Breast Cancer
}

\author{
Naoki Watanabe ${ }^{a}$ Shoko Otsuka ${ }^{a}$ Yoko Sasakia Reiko Shimojima ${ }^{a}$ Yoji Wani ${ }^{b}$ Kaori Uchino ${ }^{b}$ \\ aDepartment of Breast Surgery, ${ }^{\mathrm{b}}$ Department of Pathology, Japanese Red Cross Society Himeji Hospital Hyogo, Japan
}

\section{Keywords}

Anthracyclines - Trastuzumab - Cardiotoxicity .

Adjuvant chemotherapy · Breast cancer

\section{Summary}

Background: Retrospective analysis suggests that anthracycline-containing regimens may be superior to nonanthracycline-containing regimens in patients with human epidermal growth factor receptor 2 (HER2)-positive breast cancer. However, both trastuzumab and anthracycline have cardiotoxicity, and it remains unclear how to use trastuzumab in combination with an anthracycline to curtail their cardiotoxicity. Patients and Methods: From 2010 to 2013, we administered weekly (q1w) paclitaxel (wP) followed by $75 \mathrm{mg} / \mathrm{m}^{2}$ epirubicin, fluorouracil, and cyclophosphamide (FEC) every third week (q3w) and concurrent q1w trastuzumab $(\mathrm{H})$ to 41 patients with HER2-positive breast cancer ( $\mathrm{H}+$ group), and wP followed by FEC100 without trastuzumab to 57 patients who were HER2-negative (H- group). We routinely assessed the left ventricular ejection fraction (LVEF) by echocardiography, at the time of initiation, after WP, and after FEC, and compared them between these 2 groups. Results: LVEF decreased from 63.2 to $60.9 \%$ ( $p=0.030$ ) in the $\mathrm{H}+$ group and from 63.9 to $61.9 \%(\mathrm{p}=0.009)$ in the $\mathrm{H}$ - group. These 2 groups showed no significant difference in the reduction rate of LVEF over the period of chemotherapy (0.968 vs. 0.978 : NS, $p=0.6457)$. There was no severe cardiotoxicity or congestive heart failure in either group. Conclusion: Concurrent administration of epirubicin $\left(\mathrm{q} 3 \mathrm{w}, 75 \mathrm{mg} / \mathrm{m}^{2}\right.$ ) and trastuzumab showed no less cardiac tolerability in an adjuvant setting.

\section{Introduction}

In 2005, Buzdar et al. [1] designed a unique and tactical regimen of chemotherapy for human epidermal growth factor receptor 2 (HER2)-positive breast cancer, and performed a randomized trial for this protocol, in which they had administered anthracycline and trastuzumab concurrently. $\mathrm{He}$ achieved an outstanding pathological complete response (pCR) rate, and the patients did not experience severe adverse cardiac events. Following this report, the National Comprehensive Cancer Network (NCCN) clinical practice guideline [2] recommends the regimen: $80 \mathrm{mg} / \mathrm{m}^{2}$ paclitaxel intravenously (IV) weekly on day 1 for 12 weeks (wP) followed by FEC75 (500 mg/m² 5-fluorouracil (5-FU), $75 \mathrm{mg} / \mathrm{m}^{2}$ epirubicin, and $500 \mathrm{mg} / \mathrm{m}^{2}$ cyclophosphamide) IV cycled every 3 weeks on day 1 for 4 cycles, with concurrent administration of trastuzumab $(\mathrm{H})$ weekly $(\mathrm{wPH} \rightarrow \mathrm{FEC} 75 \mathrm{H})$ throughout this period.

However, the NCCN also added the proviso that trastuzumab should not be given at the same time as an anthracycline, except as part of the neoadjuvant trastuzumab with paclitaxel followed by FEC. The established evidence of Buzdar et al. was only based on the trial in neoadjuvant setting, but this regimen could feasibly be adopted for the adjuvant chemotherapy. We conclude that the regimen of Buzdar et al. could be treated as an exception, although the NCCN has not accepted the feasibility of concurrent use of anthracycline and trastuzumab in ground rules. However, the NCCN also commented that anthracycline-based chemotherapy regimens may be superior to non-anthracycline-based regimes in patients with HER2-positive tumors [2,3].

Over the past few years, while wPH $\rightarrow \mathrm{FEC} 75 \mathrm{H}$ may only be regarded as an exceptional option, we prefer this regimen for the treatment of HER2-positive breast cancer. This is because the regimen has some apparent advantages over the other recommended regimens in the NCCN guideline. First, the required duration is the shortest of all regimens containing

\section{KARGER \\ Fax +497614520714 \\ Information@Karger.com}

www.karger.com (c) 2014 S. Karger GmbH, Freiburg

1661-3791/14/0091-0046\$39.50/0

Accessible online at:

www.karger.com/brc
Dr. Naoki Watanabe

Department of Breast Surgery

Japanese Red Cross Society Himeji Hospital

1-12-1, Shimoteno, Himeji, Hyogo 670-8540, Japan

nawtnb@gmail.com 
both anthracycline and trastuzumab, and so contributes to shortening the duration of chemotherapy to 3-6 months, and to cutting healthcare benefit costs. Second, in the neoadjuvant setting, with this regimen trastuzumab can be administered for a longer period: 6 months from start to finish.

Before initiation of therapy, informed consent was obtained from all participants, and we ensured the feasibility of cardiac risk management. We performed echocardiography to examine the left ventricular ejection fraction (LVEF) routinely starting at initiation, and then after weekly paclitaxel, and FEC. We also concurrently administered a similar regimen, wP $\rightarrow$ FEC100, to HER2-negative breast cancer patients. To assess the cardiotoxicity of $\mathrm{wPH} \rightarrow \mathrm{FEC} 75 \mathrm{H}$, we compared the cardiac damage from the chemotherapy between these 2 groups retrospectively.

\section{Patients and Methods}

\section{Patients}

A retrospective review was performed of patients who had stage II-III breast cancer, and who had been referred to the Department of Surgery at the Japanese Red Cross Society Himeji hospital between October 2010 and April 2013. The patients received adjuvant or neoadjuvant chemotherapy. Our candidates were patients who had received an particular regimen: sequential administration of $\mathrm{wP}$ followed by FEC.

Histological confirmation of invasive tumor was performed on the specimen by core needle biopsy or by ultrasound-guided vacuum-assisted breast biopsy (Vacora ${ }^{\circledR}$ Breast Biopsy System, Medicon Inc., Osaka, Japan). The biopsy samples were also examined for HER2 overexpression by fluorescence in situ hybridization or $3+$ overexpression by immunohistochemistry (IHC). Details of methods for measuring protein overexpression by IHC and amplification by polymerase chain reaction have been previously reported [4-6]. We used antibodies (4B5, Roche Diagnostics Japan) directed against the internal domain of the HER2 protein for IHC analysis.

Estrogen and/or progesterone receptors were assessed locally on the same tissue samples. If the biopsy sample was not insufficient for IHC, we performed a pathological evaluation on the surgical specimen.

Before initiation of therapy, all patients underwent staging evaluation, which included complete history, physical examination, complete blood count $(\mathrm{CBC})$, chemistry profile, chest radiograph, and $\left[{ }^{18} \mathrm{~F}\right]$ fluorodeoxyglucose positron emission tomography (FDG-PET). Tumor size and extension were examined by contrast-enhanced magnetic resonance imaging (MRI-CE) and ultrasound sonography.

All patients were required to have adequate cardiac function in the baseline examination. They underwent an initial electrocardiogram and cardiac echogram evaluation including LVEF using a modified biplane Simpson method [7]. Patients with a history of uncompensated congestive heart failure, abnormality of the electrocardiogram, or a cardiac ejection

Table 1. Study characteristics ${ }^{\mathrm{a}}$

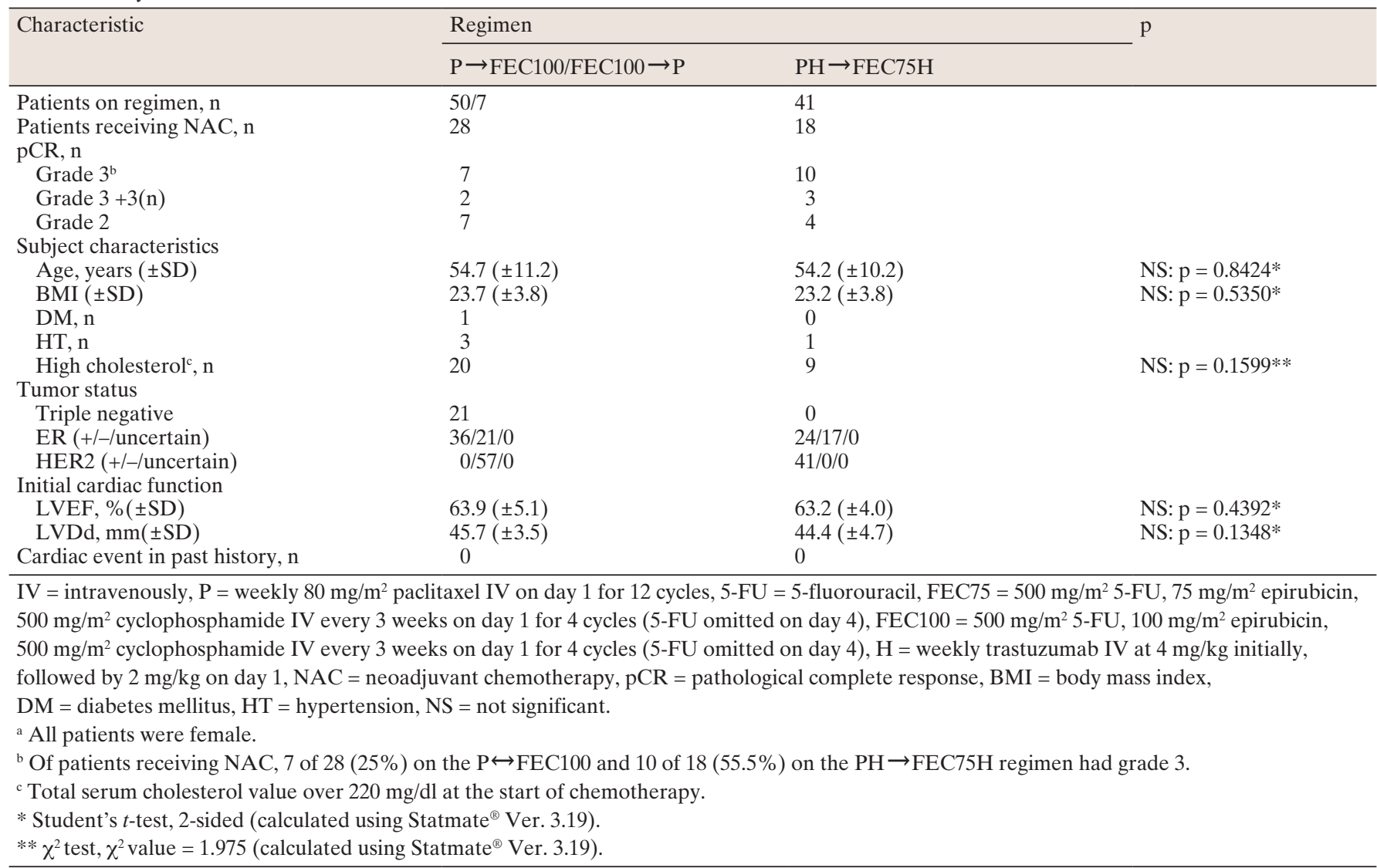


fraction less than $50 \%$ were considered not appropriate for adjuvant chemotherapy, and were excluded from this study (table 1).

\section{Chemotherapy}

In the study period, 41 patients with HER2-positive breast cancer underwent the regimen of Buzdar et al. (wPH $\rightarrow \mathrm{FEC} 75 \mathrm{H})$, i.e. concurrent weekly administration of trastuzumab (first time $4 \mathrm{mg} / \mathrm{kg}$, followed by $2 \mathrm{mg} / \mathrm{kg}$ IV on day 1 ) with wP (at $80 \mathrm{mg} / \mathrm{m}^{2} \mathrm{IV}$ on day 1 for 12 cycles) followed by FEC75 (consisting of $500 \mathrm{mg} / \mathrm{m}^{2} 5-\mathrm{FU}, 75 \mathrm{mg} / \mathrm{m}^{2}$ epirubicin, and $500 \mathrm{mg} / \mathrm{m}^{2}$ cyclophosphamide) IV repeated every 3 weeks on day 1 for 4 cycles. We modified this protocol by omitting $500 \mathrm{mg} / \mathrm{m}^{2} 5$-FU on day 4 . After sequential administration of wP and FEC, we started trastuzumab at $6 \mathrm{mg} / \mathrm{kg} \mathrm{IV}$, repeated every 3 weeks on day 1 as a single agent, with a total dose period of trastuzumab of 12 months. In the case of neoadjuvant chemotherapy, we reinitiated trastuzumab after surgery, and continued it for 6 months.

In Japan, the package leaflet for trastuzumab states that the concurrent administration of anthracycline and trastuzumab requires careful and thoughtful consideration [8]. We gained approval for the implementation of this regimen from the Institutional Review Board, which included a clinical trial scientist person from outside our company. We got informed consent from all study patients, and the administration was performed under and subject to close monitoring of cardiac function.

During the same period, 57 HER2-negative patients were administered sequential wP and FEC, without trastuzumab: 50 patients were administered $\mathrm{wP} \rightarrow \mathrm{FEC} 100$ (every 3 weeks; $500 \mathrm{mg} / \mathrm{m}^{2}$ 5-FU, $100 \mathrm{mg} / \mathrm{m}^{2}$ epirubicin, $500 \mathrm{mg} / \mathrm{m}^{2}$ cyclophosphamide) IV on day 1 for 4 cycles) and 7 were administered $\mathrm{FEC} 100 \rightarrow$ wP

The dose of paclitaxel or only epirubicin among FEC was reduced by $20 \%$ in subsequent cycles if a patient experienced grade 4 neutropenia or neutropenic fever. We administered granulocyte colony-stimulating factor until the granulocyte count exceeded 5,000/ $\mu$ l. Patients were required to have more than $1,000 / \mu$ neutrophils and more than $100,000 / \mu 1$ platelets before the administration of the next cycle of chemotherapy. The dose of drugs was also reduced if a patient experienced continuing severe nausea or vomiting of grade $\geq 3$, even after the appropriate modification of premedication.

\section{Assessments}

$\mathrm{CBC}$ and clinical chemistry were repeated at each cycle of chemotherapy, and adverse events were assessed by Common Terminology Criteria for Adverse Events (CTCAE) Ver. 4.03 [9] and documented. During FEC, CBC was not performed before the next cycle unless a patient experienced febrile neutropenia.

Patients were also observed for progressive disease in the neoadjuvant setting. Imaging studies of the tumors were performed by ultrasound sonography monthly and by MRI-CE at the initiation, and after wP and FEC. Tumor shrinkage (objective response) and disease progression were assessed using the Response Evaluation Criteria In Solid Tumors (RECIST) guidelines (version 1.1) [10]. After chemotherapy, resected specimens were evaluated by pathologists. In general, for non-palpable (clinical complete response) cases, the specimen was then sectioned into 3-5-mm slices, and was examined to assess the presence of residual microscopic disease. All axillary lymph nodes were also carefully evaluated by serial gross sectioning. 1 or 2 representative histological sections were evaluated per paraffin block. IHC staining for cytokeratin was not routinely performed on negative nodes. pCR was defined as no evidence of residual invasive cancer either in the breast or axillary lymph node.

Electrocardiograms and echocardiograms were routinely obtained at baseline, after wP, and after FEC. If conspicuous data were obtained, all were interpreted by cardiologists at our hospital. As the most commonly accepted concept in the oncology community, we defined decreased LVEF as an absolute 10-point decrease in LVEF from baseline or an LVEF of under $50 \%$ [9].

\section{Statistical Analysis}

Data for individual patients were compared using a paired, 2-tailed $t$-test, whereas data summarizing a group of patients were compared using repeated measures of analysis of variance, with $\mathrm{p}<0.05$ considered significant in all comparisons.

\section{Results}

\section{Therapeutic Performance}

Between October 2010 and April 2013, 41 patients with HER2-positive breast cancer were treated using a chemotherapy regimen containing trastuzumab $(\mathrm{H}+$ group). They were administered $\mathrm{wPH} \rightarrow \mathrm{FEC} 75 \mathrm{H} ; 18$ were treated in a neoadjuvant setting, and 23 in an adjuvant setting. During the same period, 57 HER2-negative patients, who did not receive trastuzumab, were treated (H- group); 28 were administered neoadjuvant chemotherapy, with the rest receiving adjuvant chemotherapy. They were treated with a sequential chemotherapy regimen: 50 were given wP followed by FEC100 $(\mathrm{wP} \rightarrow \mathrm{FEC100})$, and 7 FEC100 followed by wP $(\mathrm{FEC100} \rightarrow \mathrm{wP})$. Pretreatment characteristics of the patients in the 2 groups were similar, and are listed in table 1 . The number of individuals with hypercholesterolemia was larger for the H- group, but there were no significant differences. In a neoadjuvant setting, predictably, we achieved a high pathological response rate $(10 / 18,55.5 \%)$ in the $\mathrm{H}+$ group who had been treated by a trastuzumab-containing regimen.

\section{About LVEF}

The mean initial LVEF in the $\mathrm{H}+$ group was $63.2 \%$ (range 51.7-73.0, standard deviation (SD) 4.0) and in the H-group $63.9 \%$ (range 52.7-74.2, SD 5.1; not significant (NS), $\mathrm{p}=0.4392$ ). The mean initial LVDd in the $\mathrm{H}$ - group was $45.7 \mathrm{~mm}$ (range 35.8-54.3, SD 3.5), and in the H+ group $44.4 \mathrm{~mm}$ (range $36.8-52.8$, SD 4.7 ; NS, $\mathrm{p}=0.1348$ ). There was no significant difference between the initial LVEF of the 2 groups (table 1$)$.

In the $\mathrm{H}+$ group, LVEF was reduced significantly from the initial level $(63.2 \%)$ to $60.9 \%$ (SD 5.3; $\mathrm{p}=0.03022)$, and in the $\mathrm{H}$ - group from 63.9 to $61.9 \%$ (SD $4.3 ; \mathrm{p}=0.01252$ ) (fig. $1 \mathrm{~A}$, B). The reduction rate of LVEF in the $\mathrm{H}+$ group was $\times 0.968$ on average (SD 0.103 ), and in the $\mathrm{H}$ - group $\times 0.978$ on average (SD 0.101). There was no significant difference in the reduction rate of LVEF between the 2 groups $(\mathrm{NS}, \mathrm{p}=0.6457$ ) (fig. 1C). In the $\mathrm{H}+$ group, 8 in 41 cases and in the $\mathrm{H}-$ group, 17 in 57 cases showed more than $10 \%$ reduction in LVEF $\left(\chi^{2}\right.$ value $=1.335, \mathrm{p}=0.2480)($ table 2$)$. These patients received an immediate consultation with a cardiovascular medical specialist. However, all of them maintained a level of LVEF above $50 \%$. Therefore, we did not need to reduce the drug dose, and the patients completed all courses of adjuvant chemotherapy. To date, no patient of either group has experienced severe cardiotoxicity and congestive heart failure. 

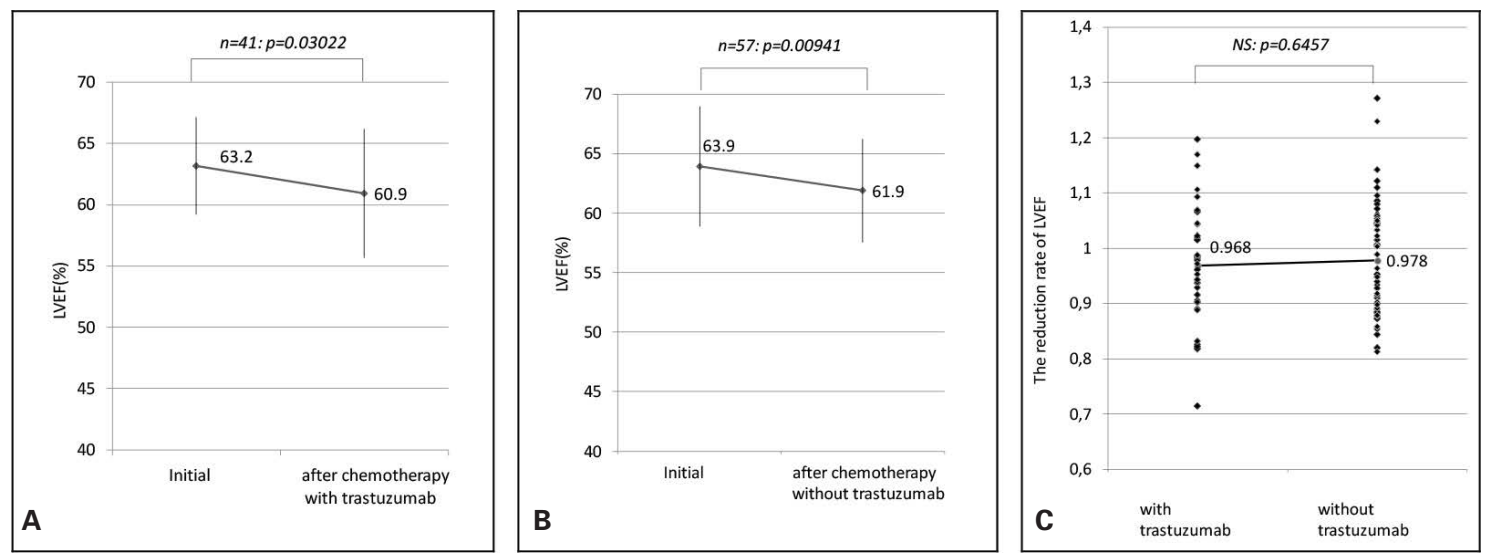

Fig. 1. A Changes in left ventricular ejection fraction (LVEF) in individual patients in the HER2-positive group, who had been administered sequential paclitaxel and FEC75, in combination with concurrent trastuzumab. B Changes in LVEF in individual patients in the HER2-negative group, who had been treated by sequential administration of paclitaxel and FEC100, but without trastuzumab. C Reduction rate of LVEF compared between the group treated by a trastuzumab-containing regimen (with trastuzumab) and the group administered paclitaxel followed by FEC, but without trastuzumab (without trastuzumab). FEC75 $=500 \mathrm{mg} / \mathrm{m}^{2} 5-\mathrm{FU}, 75 \mathrm{mg} / \mathrm{m}^{2}$ epirubicin, $500 \mathrm{mg} / \mathrm{m}^{2}$ cyclophosphamide intravenously every 3 weeks on day 1 for 4 cycles (5-FU omitted on day 4), FEC100 = 500 mg/m² 5 -FU, $100 \mathrm{mg} / \mathrm{m}^{2}$ epirubicin, $500 \mathrm{mg} / \mathrm{m}^{2}$ cyclophosphamide intravenously every 3 weeks on day 1 for 4 cycles (5-FU omitted on day 4), FU = fluorouracil.

Table 2. Adverse events

\begin{tabular}{|c|c|c|}
\hline \multirow[t]{2}{*}{ Event } & \multicolumn{2}{|l|}{ Regimen } \\
\hline & $\mathrm{P} \leftrightarrow \mathrm{FEC} 100$ & $\mathrm{PH} \rightarrow \mathrm{FEC} 75 \mathrm{H}$ \\
\hline Patients, $\mathrm{n}$ & 57 & 41 \\
\hline \multicolumn{3}{|l|}{ Cardiac safety data, $\mathrm{n}$} \\
\hline Congestive heart failure & 0 & 0 \\
\hline$>10 \%$ decrease in LV ejection fraction & 17 & 8 \\
\hline$>10 \%$ increase in LV diastolic dimension measurement & 5 & 9 \\
\hline \multicolumn{3}{|l|}{ Other adverse events, $\mathrm{n}$} \\
\hline Grade 4 neutropenia ${ }^{a}$ & 6 & 1 \\
\hline Grade 3 neutropenia & 4 & 3 \\
\hline Neutropenic infections & 2 & 1 \\
\hline Hospitalization due to febrile neutropenia & 0 & 0 \\
\hline Chemotherapy dose reduction as a result of neutropenia & 6 & 1 \\
\hline Chemotherapy dose reduction as a result of grade 2 nausea & 3 & 0 \\
\hline Allergic reactions, no therapy modification & 0 & 1 \\
\hline ALT/AST increased, grade $1 / 2$ & $17 / 1$ & $14 / 3$ \\
\hline
\end{tabular}

$\mathrm{LV}=$ left ventricular, $\mathrm{ALT}=$ alanine aminotranferease, $\mathrm{AST}=$ aspartate aminotransferase.

a Peripheral blood cell count examined every 3 weeks, at time of chemotherapy.

We compared the LVEF changes during different periods of the chemotherapy looking at values at initiation, and those after paclitaxel, and after FEC in the $\mathrm{H}+$ group and the $\mathrm{H}-$ groups (fig. 2A). In the $\mathrm{H}-$ group, since 7 patients were treated in the order $\mathrm{FEC} \rightarrow \mathrm{wP}$, the data shown in figure 2 are for the remaining 50 patients. In the $\mathrm{H}+$ group, the LVEF was severely reduced during the $\mathrm{wPH}$ period compared to the FEC75H period, and the LVEF returned toward the baseline during FEC75H. In both group, the LVEF was reduced more in the former paclitaxel-dosing phase than in latter FEC phase (fig. 2B). The reduction rate of LVEF, hence, got worse in the following order: later FEC75H, later FEC100, earlier wP, and earlier wPH.

\section{Other Adverse Event}

None of our patients experienced grade 4 neutropenia during the paclitaxel phase of the therapy. Between each FEC administration, unless a patient returned complaining of neutropenic fever, we could not check the peripheral blood cell count, and so could not check whether any adverse events occurred for the 3 weeks up to the next administration. Considering that caveat, only 2 patients had neutropenic fever in the FEC100 phase, and 1 in the FEC75H phase (table 2). The dose of epirubicin was reduced by $20 \%$ in those cases. Beside these 2 cases with febrile neutropenia, 4 cases had a dose reduction because of grade 4 neutropenia, and 3 because of grade 2 nausea during the FEC100 phase of chemotherapy. Grade 1 or 2 elevation of alanine aminotranferease (ALT) or aspartate aminotransferase (AST) was seen in the patients in the paclitaxel phase whether with or without trastuzumab. 

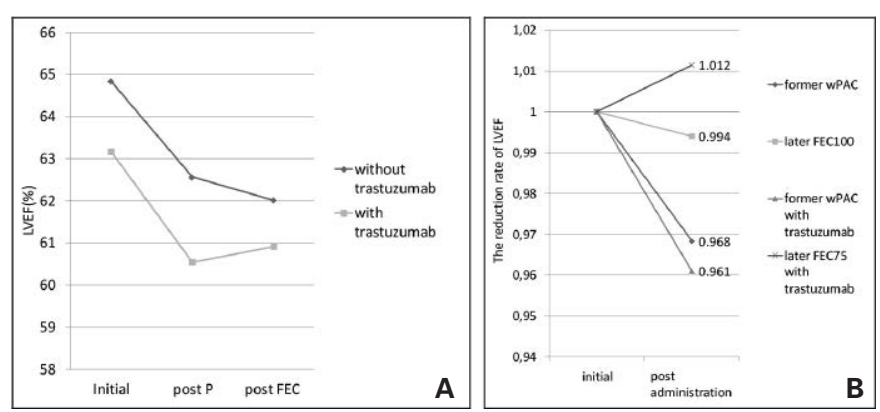

Fig. 2. A We compared the LVEF at initiation, after paclitaxel administration (post-paclitaxel), and after FEC administration (post-FEC) between the HER2-positive group $(n=41)$ and the HER2-negative group $(n=50)$. The patients of both groups were administered paclitaxel followed by FEC sequentially, and the former were concurrently administered trastuzumab throughout this period, while the latter received no trastuzumab. B Reduction rate of LVEF compared between the 4 periods treated by a trastuzumab-containing regimen, earlier paclitaxel phase and later FEC75 phase, and without trastuzumab regimen, earlier paclitaxel phase and later FEC100.

1 patient had an allergic reaction at the initial administration of the paclitaxel. Transient redness was seen her in face, and she complained of temporary headache. She recovered from these symptoms as soon as we decreased the infusion rate of paclitaxel.

\section{Discussion}

The regimen of Buzdar et al. [1], i.e. weekly paclitaxel followed by FEC with concurrent trastuzumab, is completely unique among the NCCN recommendations. According to the proviso given by the NCCN, however, it was exceptional because the anthracycline, epirubicin, is used with trastuzumab concurrently in this protocol [2]. This reflects a certain fear of clinical practitioners against the synergistic cardiotoxicity caused by the combination use of anthracycline and trastuzumab.

Russell et al. [11] reviewed the combined cardiac data from the NSABP B-31 and NCCTG N9831 clinical trials, and found that trastuzumab-treated patients had a $2.0 \%$ incidence of symptomatic heart failure events compared with $0.45 \%$ in the chemotherapy-alone arm. Up to now, the cardiotoxicity of epirubicin has been studied almost exclusively, and was proven to be low in comparison with optimal dosing of doxorubicin [12-15]. However, these 2 trials were based on doxorubicin, which was not administered concurrently with trastuzumab.

In the HERA study [16], severe congestive heart failure occurred in more patients on trastuzumab than in the observation group. Heart failure (NYHA III and IV) occurred in $0.6 \%$ in the trastuzumab arm (vs. standard arm: $0 \%$ $\mathrm{p}<0.0001$ ), and a significant decrease of LVEF of $3 \%$ oc- curred (vs. standard arm: $0.5 \% \mathrm{p}<0.0001$ ). In that study, the type of adjuvant chemotherapy was a doxorubicin-containing regimen for $23.4 \%$, and an epirubicin-based regimen for $44.5 \%$. However, in this trial, trastuzumab was also administered sequentially to the other drug. These large trials have not shown that the concurrent use of trastuzumab and anthracyclines would enhance the cardiotoxicity more synergistically than their sequential use. We agree that their concurrent use can be hazardous.

Buzdar et al. [1] showed that FEC75 could be administered safely without severe cardiotoxicity with trastuzumab in neoadjuvant setting. As expected, we were also able to perform the regimen of Buzdar et al. feasibly in adjuvant setting. We followed all patients for more than 1 year after the completion of chemotherapy and assessed their cardiac function routinely. The LVEF in both group recovered uneventfully. In the group receiving $\mathrm{wPH} \rightarrow \mathrm{FEC} 75 \mathrm{H}$, the average of LVEF recovered from 60.9 to $61.5 \%(\mathrm{n}=25)$, and in the group receiving $\mathrm{wP} \rightarrow \mathrm{FEC100}$, from 61.9 to $62.4 \%(\mathrm{n}=24)$. This means that, as long as we selected an adequate type of anthracycline, its feasible dose, and an appropriate schedule, we could administer a concurrent regimen of anthracycline and trastuzumab, while avoiding adverse cardiac events, thus shortening the total duration of chemotherapy by a few months.

To select regimens that do not contain an anthracycline is a good notion for minimizing the risk of cardiac toxicity. However, retrospective analyses of the correlation of HER2 overexpression and chemotherapy response have suggested that anthracyclines play an important role in the management of HER2-overexpressing breast cancer [4, 17-20]. Recently, BCIRG 006 reported an attractive regimen for HER2-positive breast cancer that consists of docetaxel, carboplatin, and trastuzumab (TCH) and does not contain anthracyclines [21]. However, TCH could not demonstrate better efficacy outcomes than an anthracycline-containing regimen: doxorubicin and cyclophosphamide followed by docetaxel and trastuzumab (AC-TH). Disease-free survival (DFS) and overall survival (OS) for AC-TH and $\mathrm{TCH}$ were $92.6 \%$ and $89.7 \%$, and $97.5 \%$ and $96.2 \%$, respectively. Although not significant, $\mathrm{TCH}$ seems to be inferior to the anthracycline-containing regimen.

Anthracyclines remain essential for treating HER2-positive breast cancer patients. Therefore, to mitigate the cardiac toxicity, we have to devise the best protocol to use trastuzumab in combination with anthracycline. Further questions arise: Which type of anthracycline should be used, doxorubicin or epirubicin? Which dose has better tolerability, FEC100 or FEC75? Should we administer anthracycline and trastuzumab concurrently or sequentially?

In figure $2 \mathrm{~B}, \mathrm{LVEF}$ dropped considerably during the $\mathrm{wPH}$ phase, but recovered in the anthracycline-containing $\mathrm{FEC75H}$ phase, even with dosing trastuzumab concomitantly throughout a period of chemotherapy. Contrary to our expectation, 
the LVEF was reduced more in the former wP phase in all patients. This suggests that cardiac function is impaired mainly at the introduction of the chemotherapy.

Cardiotoxicity caused by trastuzumab is most likely secondary to inhibition of cardiomyocyte HER2, also known as ErbB2 signaling, thereby interfering with normal growth, repair, and survival of cardiomyocytes [22-24]. AC, FEC100, or heavy administration of anthracyclines as the introduction of adjuvant chemotherapy, even without concurrent trastuzumab, may thereby cause severe cardiomyocyte damage, and subsequent trastuzumab with wP may inhibit their repair. We have no practical experience of the regimen of anthracycline without trastuzumab followed by taxane with trastuzumab, but figure $2 \mathrm{~B}$ shows that the protocol $\mathrm{FEC100} \rightarrow \mathrm{wPH}$ may result in a worse LVEF drop. At any rate, to mitigate the cardiac damage, the dosing regimen in the order $\mathrm{wPH} \rightarrow \mathrm{FEC} 100$ may be safer in the combined use with trastuzumab.

Our study was limited by the retrospective nature of the available data, and needs more long-term follow-up. Never- theless, all patients completed the regimen uneventfully through the period of the chemotherapy, and showed no less cardiac tolerability of the regimen $w \mathrm{PH} \rightarrow \mathrm{FEC} 75 \mathrm{H}$ in comparison with $\mathrm{wP} \rightarrow \mathrm{FEC100}$. Our study shows that attention should be paid not only to whether the administration is concurrent or sequential, but also to the rotation and selected type and dose of anthracyclines in the combination with trastuzumab. As it turned out, the regimen of Buzdar et al., wPH followed by $\mathrm{FEC} 75 \mathrm{H}$, is well designed, and one of the best choices for performing adjuvant chemotherapy containing anthracycline and trastuzumab in combination.

\section{Disclosure Statement}

The authors declare no conflict of interest and disclose no financial and personal relationships with other people or organizations that could inappropriately influence/bias this work.

\section{References}

1 Buzdar AU, Ibrahim NK, Francis D, et al.: Significantly higher pathologic complete remission rate after neoadjuvant therapy with trastuzumab, paclitaxel, and epirubicin chemotherapy: Results of a randomized trial in human epidermal growth factor receptor 2-positive operable breast cancer. J Clin Oncol 2005;23:3676-3685.

2 NCCN: Clinical Practice Guidelines in Oncology, in: Theriault RL, Carlson RW, Allred C, et al (eds): Breast Cancer, Version 3.2013. National Comprehensive Cancer Network 2013.

3 Siegel R, Naishadham D, Jemal A: Cancer statistics, 2012. CA Cancer J Clin 2012;62:10-29.

4 Muss HB, Thor AD, Berry DA, et al.: c-erbB-2 expression and response to adjuvant therapy in women with node-positive early breast cancer. N Engl J Med 1994;330:1260-1266.

$\checkmark 5$ Thor AD, Berry DA, Budman DR, et al.: erbB-2, p53, and efficacy of adjuvant therapy in lymph node-positive breast cancer. J Natl Cancer Inst 1998;90:1346-1360.

6 Neubauer A, Neubauer B, He M, et al.: Analysis of gene amplification in archival tissue by differential polymerase chain reaction. Oncogene 1992;7:10191025.

7 LeJemtel TH, Sonnenblick EH, Frishman WH: Diagnosis and manegement of heart failure, in: Fuster V, Alexander R, O`Rourke RA, et al. (eds): Hurst's The Heart, 10th ed. New York, McGrawHill, 2001, pp 687-724.

8 Chugai Pharmaceutical Co., Ltd. 2012.

9 Common Terminology Criteria for Adverse Events (CTCAE), version 4.03. National Cancer Institute 2009.
10 Eisenhauer EA, Therasse P, Bogaerts J, et al.: New response evaluation criteria in solid tumours: Revised RECIST guideline (version 1.1). Eur J Cancer 2009;45:228-247.

11 Russell SD, Blackwell KL, Lawrence J, et al.: Independent adjudication of symptomatic heart failure with the use of doxorubicin and cyclophosphamide followed by trastuzumab adjuvant therapy: A combined review of cardiac data from the National Surgical Adjuvant Breast and Bowel Project B-31 and the North Central Cancer Treatment Group N9831 clinical trials. J Clin Oncol 2010;28:3416-3421.

12 Kaklamani VG, Gradishar WJ: Epirubicin versus doxorubicin: Which is the anthracycline of choice for the treatment of breast cancer? Clin Breast Cancer 2003;4 Suppl 1:S26-33.

13 Stohr W, Paulides M, Brecht I, et al.: Comparison of epirubicin and doxorubicin cardiotoxicity in children and adolescents treated within the German Cooperative Soft Tissue Sarcoma Study (CWS). J Cancer Res Clin Oncol 2006;132:35-40.

14 Farolfi A, Melegari E, Aquilina M, et al.: Trastuzumab-induced cardiotoxicity in early breast cancer patients: A retrospective study of possible risk and protective factors. Heart 2013;99:634-639.

15 Khasraw M, Bell R, Dang C. Epirubicin: Is it like doxorubicin in breast cancer? A clinical review. Breast 2012;21:142-149.

16 Smith I, Procter M, Gelber RD, et al.: 2-year follow-up of trastuzumab after adjuvant chemotherapy in HER2-positive breast cancer: A randomised controlled trial. Lancet 2007;369:29-36.

17 Cooke T, Reeves J, Lanigan A, Stanton P: HER2 as a prognostic and predictive marker for breast cancer. Ann Oncol 2001;12 Suppl 1:S23-28.
8 Di Leo A, Larsimont D, Gancberg D, et al.: HER-2 and topo-isomerase IIalpha as predictive markers in a population of node-positive breast cancer patients randomly treated with adjuvant CMF or epirubicin plus cyclophosphamide. Ann Oncol 2001;12:1081-1089.

19 Harris LN, Yang L, Liotcheva V, et al.: Induction of topoisomerase II activity after ErbB2 activation is associated with a differential response to breast cancer chemotherapy. Clin Cancer Res 2001;7: 1497-1504.

20 Petit T, Borel C, Ghnassia JP, et al.: Chemotherapy response of breast cancer depends on HER-2 status and anthracycline dose intensity in the neoadjuvant setting. Clin Cancer Res 2001;7:15771581.

21 Valero V, Forbes J, Pegram MD, et al.: Multicenter phase III randomized trial comparing docetaxel and trastuzumab with docetaxel, carboplatin, and trastuzumab as first-line chemotherapy for patients with HER2-gene-amplified metastatic breast cancer (BCIRG 007 study): Two highly active therapeutic regimens. J Clin Oncol 2011;29:149-156.

22 Ewer MS, O‘Shaughnessy JA: Cardiac toxicity of trastuzumab-related regimens in HER2-overexpressing breast cancer. Clin Breast Cancer 2007;7: 600-607.

23 Crone SA, Zhao YY, Fan L, et al.: ErbB2 is essential in the prevention of dilated cardiomyopathy. Nat Med 2002;8:459-465.

24 Ozcelik C, Erdmann B, Pilz B, et al.: Conditional mutation of the ErbB2 (HER2) receptor in cardiomyocytes leads to dilated cardiomyopathy. Proc Natl Acad Sci USA 2002;99:8880-8885. 